\title{
Preparation and Characterization of novel PSf/PVP/PANI-nanofiber Nanocomposite Hollow fiber Ultrafiltration Membranes and their possible applications for Hazardous Dye rejection
}

\author{
Avin J. Kajekar ${ }^{\mathrm{a}}$, B.M. Dodamani ${ }^{\mathrm{a}}$, Arun M. Isloor ${ }^{\mathrm{b},}{ }^{*}$ Zulhairun Abdul Karim ${ }^{\mathrm{c}}, \mathrm{Ng} \mathrm{Be}$ \\ Cheer $^{\mathrm{c}}$, A. F. Ismail ${ }^{\mathrm{c}}$ and Simon J. Shilton ${ }^{\mathrm{d}}$ \\ ${ }^{a}$ Department of Applied Mechanics and Hydraulics, National Institute of Technology \\ Karnataka, Surathkal, Mangalore 575 025, India. \\ ${ }^{\mathrm{b}}$ Advanced Membrane TEchnology Research Centre (AMTEC), Universiti Teknologi \\ Malaysia, 81310 UTM, Skudai, Johor, Malaysia. \\ ${ }^{\mathrm{c}}$ Membrane Technology Laboratory, Department of Chemistry, National Institute of \\ Technology Karnataka, Surathkal, Mangalore 575 025, India. \\ ${ }^{\mathrm{d}}$ Department of Chemical and Process Engineering, University of Strathclyde, James \\ Weir Building, 75 Montrose Street, Glasgow, G1 1XJ, Scotland
}

\begin{abstract}
In the present study, PANI (Polyaniline)-nanofibers were synthesized by interfacial polymerization technique, dispersed in n-Methyl-2-Pyrrolidone (NMP) solvent and blended with PVP (Polyvinylpyrrolidone)/PSf (Polysulfone) for preparing the novel hollow fiber membrane by dry-wet spinning technique. The newly prepared nanocomposite ultrafiltration hollow fiber membrane is characterized by Scanning Electron Microscope (SEM), Contact Angle, Zeta Potential and Differential Scanning Calorimeter (DSC). Filtration studies are conducted to measure the membrane Pure Water Flux (PWF), rejection of Hazardous Dye (Reactive Red 120) and Fouling Resistance. The maximum rejections are obtained for M 0.5 membrane with $99.25 \%$ rejection of RR120 hazardous dye at 2 bar pressure. The pure water flux, percentage rejection, antifouling property and thermal resistance increased with increase in PANI-nanofiber concentration. The contact angle of the membrane decreased with increasing PANI-nanofiber concentration, which indicated increased hydrophilicity of the new membranes.
\end{abstract}

Keywords: Hollow fiber membrane, Ultrafiltration, PANI-nanofiber, Dye rejection

*Corresponding author: isloor@yahoo.com, Tel: +919448523990 


\section{Introduction}

Water is the most important resource on this planet. It is the basic necessity for survival of all living organisms and is used in agriculture and most of the industries. Among the chemical industries, dye industry is also one among them. Dye and its intermediates are used in textile, printing inks, paper, plastics, paint and food industries to add color and patterns [1]. Nowadays, natural dyes are being replaced by synthetic chemical dyes, as they are more economical, have brighter colours, good retaining capacity and are easy to apply on the fabrics. There are more than 100,000 commercial dyes and their total consumption in the worldwide textile industry is more than 10,000 tonnes/year and this in turn discharges more than 100 tonnes/year of dyes into waste streams [2]. The water used for processing dyes, rinsing textiles and cleaning the processing equipment become highly contaminated and their discharge inturn pollutes the surface and ground water. Dyes have complex aromatic molecular structure and because of their synthetic origin, they are very stable to temperature, oxidizing agents and bio-degradation. Thus, they are a grave threat to human, animal and aquatic life due to their non-degradable nature, undesirable color, toxic and carcinogenic byproducts and their capacity to increase Chemical oxygen demand (COD) levels of water sources [1].

Various methods such as, adsorption [2-3], flocculation-coagulation [4], advanced oxidation processes [5-6] and membrane separation [7-14] have been developed and adapted for removal of the dye and its intermediates from the contaminated water. Membrane filtration technologies are considered as one of the most effective for removal of contaminants from water due to their high efficiency, selective removal, easy operation and small footprint. Some of the membrane processes used are, reverse osmosis (RO), nanofiltration (NF), ultrafiltration (UF), membrane electrolysis and membrane bio-reactor (MBR).

Ong et al. [7] have evaluated the performance of membranes under various operating conditions for both lab-scale as well as pilot-scale and reported very robust performance with more than 90\% rejection for various dyes. Yu et al. [8] and Wei et al. [9] have used thin-film composite hollow fiber nanofiltration for successful removal of dyes from aqueous solutions with maximum rejections of greater than 99\%. Zheng and team [10] prepared positively 
charged thin-film composite hollow fiber nanofiltration membranes and observed rejections of $99.8 \%, 99.8 \%$ and $99.2 \%$ for Brilliant Green, Victoria Blue B and Crystal Violet respectively using the submerged filtration technique. Maurya et al. [11] successfully used Polysulfone-Polyvinlypyrrolidone ultrafiltration hollow fiber membranes for treatment of aqueous dye solutions such as Rhodamine B and Reactive Black-5 and observed a maximum dye rejection of $97 \%$ along with a maximum flux of $35 \mathrm{~L} \cdot \mathrm{m}^{-2} \cdot \mathrm{h}^{-1}$. Afonso and Borquez [12] have adopted the ultrafiltration technique for treatment of seafood processing wastewaters and have shown that UF can be a promising technique for recovery of valuable proteins. Hamid et al. [13] have prepared $\mathrm{PSf} / \mathrm{TiO}_{2}$ hollow fiber ultrafiltration membranes for the removal of humic acid and achieved rejection greater than $91 \%$ along with less fouling sensitivity to humic acid deposition. Yuliwati et al. [14] used PVDF based hollow fiber ultrafiltration membranes for treatment of refinery wastewater and reported flux of 82.5 L. $\mathrm{m}^{-2} \cdot \mathrm{h}^{-1}$ and maximum rejection of $98.8 \%$.

Ultrafiltration is a well-established membrane technique and has been used for many applications. Ultrafiltration requires much lesser energy than nanofiltration and or reverse osmosis.. Ultrafiltration separation based on size exclusion or particle capture. Ultrafiltration can be applied in either cross-flow or dead-end mode. Adapting the cross-flow mode for dye removal from aqueous medium in addition to higher resistance to scale formation, has an added advantage that, it separates out the raw wastewater into purified water and a dye concentrate stream. This concentrate stream can be further processed to recycle and reuse salts and dyes and thus mitigate environmental pollution. In membrane based treatment of dye wastewater, there are multiple retentate streams which can be reused within the dyeing industry. Recovery of water and sodium chloride up to $99 \%$ has been reported by Allègre et al. [15] [16]. The recovered water and salts have been successfully reused within the dyeing industry. Few other case studies [17] also report recovery of either water or added salts for reuse in the dye industries.

Recovery of dyes from wastewater are usually more difficult as most dyeing operations use many different colors and it may not be feasible to separate individual dyes to reuse. A textile plant in Liberty, South Carolina has reported a method of recovering indigo dye from the rinse water since 1981 [18]. Typically the process needs to be isolated before adding other dyes and an ultrafilter is used to recover the indigo dye at the process where it is generated. This has yielded considerable cost savings from recovering the expense indigo dye and the cost for the dye recovery system was recovered before the end of its second year of operation [18]. It is reported that a project sponsored by the Environmental Protection Agency (EPA) 
has also set up a process for reclamation and reuse of hot rinse water and neural, anionic and cationic dyes [19].

Polysulfone (PSf) membranes have high mechanical strength along with good thermal and chemical resistance and hence have been widely used for many applications. It is widely available, inexpensive, easy to process and very stable. However, its hydrophobic nature reduces its water flux and fouling resistance, which lessens its applications for water filtration. Hence various techniques have been used to improve its porosity and surface hydrophilicity like, grafting, polymer blending, coating and irradiation [20]. Polymer blending technique for hydrophilic surface modification is one of the preferred options due to its good performance, easy operation and stability of the product. Isloor and co workers prepared novel PSf membranes for various applications using different additives like chitosan and its derivatives [21-22], poly-amides [23], $\mathrm{TiO}_{2}$ nanotubes [24] etc., and have seen increased performance and fouling resistance.

Nanocomposite membranes have gained a lot of interest due to the significant improvement of membrane permeability, increased flux, hydrophilicity and antifouling property [25]. Nanomaterials like $\mathrm{TiO}_{2}$ nanoparticles, $\mathrm{Al}_{2} \mathrm{O}_{3}$ nanoparticles, single and multiwalled Carbon nanotubes, have been successfully blended with polymer materials to produce membranes having varied and unique properties. Razmjou et al. [26] studied performance of polyethersulfone nanocomposite membranes by incorporating $\mathrm{TiO}_{2}$ nanoparticles and have observed enhancement in pure water flux, higher thermal resistance and larger pore size. Nanofiber incorporated membranes are expected to remove particles from the aqueous phase at a high rejection rate without significant fouling and have been proposed to be used for pre-treatment for reverse osmosis [25]. Nanocomposite membranes are observed to have increased membrane surface hydrophilicty, water permeability and fouling resistance. Antimicrobial nanomaterials such as nano-Ag and Carbon nano tubes (CNT) have been incorporated to inhibit biofilm formation. Jeong et al. [27] prepared thin film nanocomposite membranes for reverse osmosis application using zeolite nanoparticles dispersed within polyamide films and has reported increased permeability with higher zeolite nanoparticle loading where rejection was in excess of $90 \%$ at the $50 \%$ recovery and was comparable to commercial RO membranes.

Polyaniline (PANI) is an organic conducting polymer, which has been used for a wide variety of applications. It is also very easy to synthesize and has good environmental stability. It has been used in ultrafiltration membranes, proton exchange membranes, gas separation and pervaporation membranes for many applications. PANI nanomaterials have been 
synthesized in various morphologies like nanoparticle, nanotube, nanoflake and nanofiber and used in polymer blends [28]. Some of the different methods used to fabricate PANInanofibers are hard and soft templates, interfacial polymerization, seeding, rapid mixing and electrospinning. PANI-nanofiber exhibits varied properties when compared to bulk PANI, which has increased surface area and better processibility [29]. Better processibilty relates to better dispersion and easier bonding during membrane formation. Polymer nanofibrous membranes are also reported to have many attractive attributes to increase membrane permeability [30]. Fan et al. [30] have shown that, the addition of PANI-nanofibers have resulted in improved membrane performance due to increased porosity and interconnected pores within the membrane structure.

Polyvinylpyrrolidone (PVP) is a water-soluble polymer and has been used as a poreforming agent for membranes. Chakrabarty et al. [31] demonstrated that, the addition of PVP to membrane casting solutions results in the increase of membrane porosity as well as increase in the number of pores.

Hollow fiber membranes have been used in several commercial applications such as bio-separations, water purification, wastewater treatment and gas separations due to their excellent mass-transfer properties. Hollow fiber membranes have higher surface area per unit of membrane module volume and hence increased water flux [7]. For preparation of the hollow fiber membranes, the parameters during spinning are very important and these are to be carefully controlled to prepare membranes of required characteristics for specific applications. Some of these parameters are the type of polymers, solvents used, additives added to the polymer dope, the rate of extrusion of dope solution and bore fluid, type of bore fluid, the air gap distance, and the type of coagulant and coagulant bath temperature [32].

In view of the above observations and in order to enhance the membrane properties, it was planned to combine the properties of these individual components. In the present study, PANI-nanofibers were synthesized by interfacial polymerization technique wherein the polymerization reaction takes place at the interface of the two immiscible liquids. The PANInanofibers are then dispersed in n-Methyl-2-Pyrrolidone (NMP) solvent and then blended with PVP and PSf for preparing the novel hollow fiber membranes by dry-wet spinning technique. The newly prepared nanocomposite ultrafiltration hollow fiber membrane is then characterized by Scanning Electron Microscope (SEM), Contact Angle, Zeta Potential and Differential Scanning Calorimeter (DSC). Filtration studies are conducted to measure the membrane Pure Water Flux (PWF), rejection of hazardous dye (Reactive Red 120) and flux recovery for the membranes. To the best of our knowledge this is the first study on the effect 
of PANI-nanofibers on hollow fiber ultrafiltration membranes and their possible applications for rejection of Reactive Red 120 dye from water.

\section{Materials and Methods}

\subsection{Materials}

Udel ${ }^{\circledR}$ P-1700 Polysulfone (PSf) pellets were obtained from Solvay company. Aniline was purchased from AnalaR Normapur ${ }^{\circledR}$. 1N Hydrochloric Acid ( $\left.\mathrm{HCl}\right)$, n-Methyl-2-Pyrrolidone (NMP) and Ammonium Persulfate (APS) was purchased from Merck. Polyvinylpyrrolidone (PVP) and Reactive Red 120 (RR120) dye were procured from Sigma-Aldrich. The Molecular structure of RR120 is shown in Fig.1. O-Xylene was purchased from S.D FineChem Ltd. Aniline was distilled under vacuum before usage and all other chemicals were used as received.

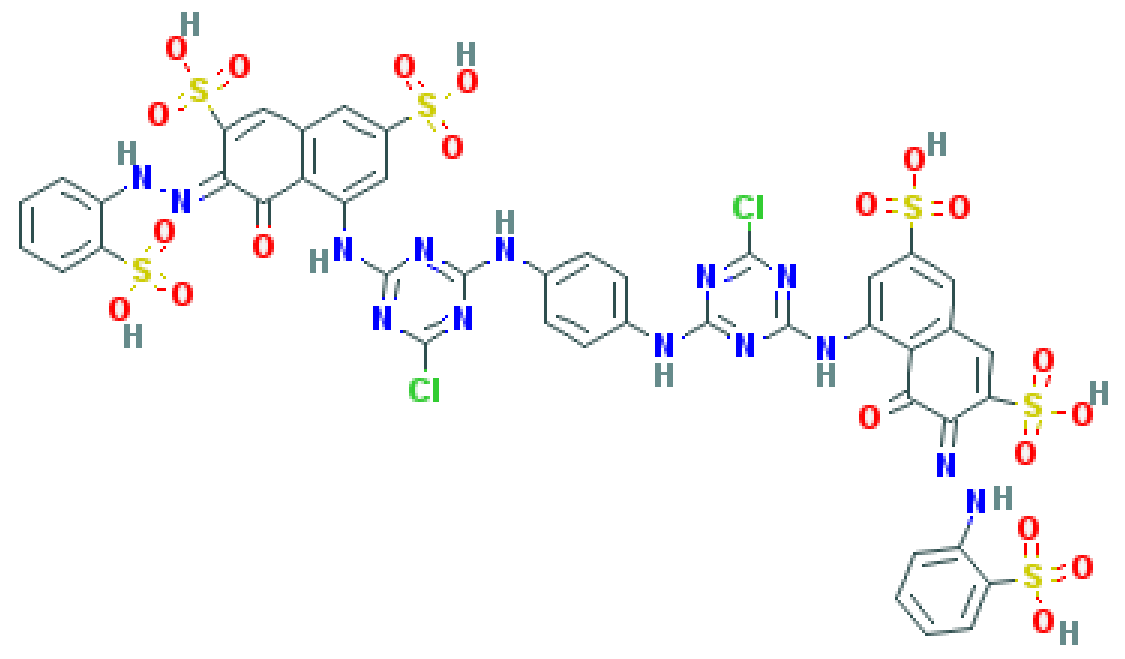

Fig.1: Molecular structure of Reactive Red 120 dye.

\subsection{Synthesis of PANI-nanofibers}

PANI-nanofibers were synthesized by interfacial polymerization of aniline as reported in literature [33]. The molar ratio of Aniline to APSwas kept at 1:1. Thus, $2 \mathrm{~g}$ of Aniline was dissolved in $2 \mathrm{ml}$ of o-Xylene and $4.674 \mathrm{~g}$ of APS was dissolved in $98 \mathrm{ml}$ of $1 \mathrm{~N} \mathrm{HCl}$ by stirring. The aniline solution was added slowly to the APS solution with vigorous stirring. The reaction is allowed to continue for 120 minutes and the blue-green PANI-nanofiber precipitate was collected. The precipitated product is then washed with deionized water and methanol continuously and separated by centrifuging. The PANI-nanofiber product is then dried in oven for 48 hours and ground into a fine powder using mortar and pestle. 


\subsection{Preparation of PSf/PVP/PANI-nanofiber dopes}

The polymer solution used for spinning hollow fiber membrane is termed as 'dope'. PANInanofibers in various concentrations are added to NMP solvent and sonicated for 30 minutes for effective dispersion. After this, PVP and PSf are dissolved in NMP under heating and continuous stirring for 48 hours until a homogeneous solution is formed. For all the prepared solutions, mass ratio of PSf to total solution was 15 weight $\%$ and PVP was 2 weight $\%$ of PSf. The PANI-nanofiber concentrationvaried between $0-1.0$ weight $\%$ of PSf and the rest was made up with NMP as detailed in Table1.

Table1: Dope solutions for PSf/PVP/PANI Nanofiber hollow fiber membrane

\begin{tabular}{|c|c|c|c|c|}
\cline { 2 - 5 } \multicolumn{1}{c|}{} & PSf (g) & PVP (g) & PANI-nf (g) & NMP (g) \\
\hline M0 & 18 & 0.36 & 0.0 & 81.64 \\
\hline M1 & 18 & 0.36 & 0.045 & 81.60 \\
\hline M2 & 18 & 0.36 & 0.090 & 81.55 \\
\hline M3 & 18 & 0.36 & 0.180 & 81.46 \\
\hline
\end{tabular}

\subsection{Preparation of PSf/PVP/PANI-nanofiber hollow fiber membranes}

The novel membrane is spun by dry-wet spinning method at room temperature by hollow fiber spinning machine as shown in Fig.2.

(a)

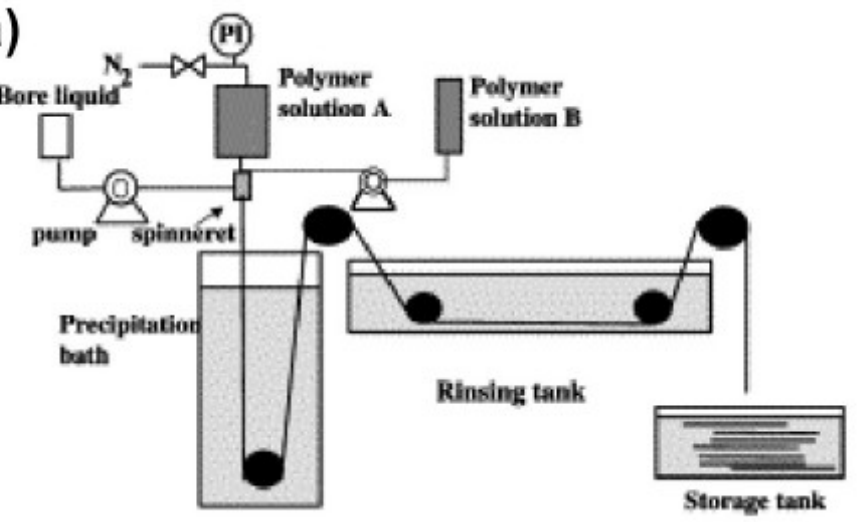

(b)

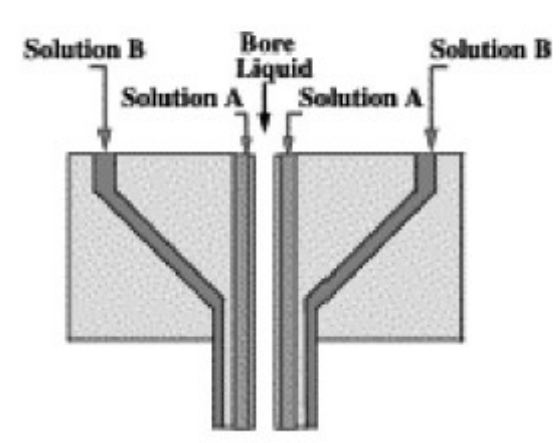

Fig.2: (a) Schematic representation of the hollow fiber spinning set up, (b) cross-section of the spinneret during spinning [24].

The polymer dope solution was pressurized through a spinneret by controlling the extrusion rate. The spinneret was placed at a fixed distance above the water bath for the dry-wet process. Distilled water was used as a bore fluid to create the hollow fiber. The hollow fiber that emerged from the tip of the spinneret was guided through the two water baths and carefully adjusted to match the free falling velocity. The fibers were then finally guided to a 
collection drum after the solidification process. The detailed spinning parameters are listed in Table 2.

Table 2: Spinning Parameters for PSf/PVP/PANI-nanofiber hollow fiber membranes

\begin{tabular}{|l|c|}
\hline \multicolumn{1}{|c|}{ Parameter } & Value \\
\hline Dope Extrusion Rate & $2.5 \mathrm{ml} / \mathrm{min}$ \\
\hline Bore Fluid & Distilled Water \\
\hline Bore Fluid Rate & $0.833 \mathrm{ml} / \mathrm{min}$ \\
\hline Collection Drum Rate & $9.4 \mathrm{~m} / \mathrm{min}$ \\
\hline Air gap distance & $5 \mathrm{~mm}$ \\
\hline Spinneret o.d/i.d & $0.3 / 0.6 \mathrm{~mm}$ \\
\hline
\end{tabular}

\subsection{Post-Treatment of PSf/PVP/PANI-nanofiber membranes}

The newly spun hollow fibers were immersed in distilled water bath for 3 days, with daily change of the water to remove residual solvent. The hollow fibers were then post-treated with $10 \mathrm{wt} . \%$ glycerol aqueous solution as a non-solvent exchange for 1 day to minimize fiber shrinkage and pore collapse [14]. Finally the treated fibers were dried for 3 days and then they were used to prepare hollow fiber test modules.

\subsection{Membrane Characterization}

\subsubsection{Morphology of the membrane}

The surface and cross section morphologies of the membrane were studied using a table top Scanning Electron Microscope (SEM) (Model: TM 3000, Hitachi). The hollow fiber membranes were immersed in liquid nitrogen and fractured to obtain its cross section. All samples were gold sputtered under vacuum before the SEM measurements.

\subsubsection{Membrane Contact Angle}

To evaluate the membrane surface hydrophilicity, the static contact angle was measured by sessile drop method using contact angle goniometer (Model: OCA 15EC, Dataphysics) [34]. At least twenty water contact angles at different locations on membrane surface were averaged and reported.

\subsubsection{Zeta Potential measurements}

Zeta Potential was calculated after measuring the streaming potentials of the hollow fiber membranes. To study the nature of membrane surface charge between $\mathrm{pH}$ ranges of 2.0-7.0, a multi-point testing approach was adopted using a SurPASS electrokinetic analyser (Anton Paar, Graz, Austria). For the analysis, potassium chloride $(1 \mathrm{mM})$ was used as electrolyte and 
0.1M Hydrochloric acid and $0.1 \mathrm{M}$ sodium hydroxide were used as titration solutions. All Zeta Potential calculations were the average of four measurements at the same $\mathrm{pH}$.

\subsubsection{Differential Scanning Calorimetry studies}

Differential Scanning Calorimetry studies help us to measure the glass transition temperature $\left(\mathrm{T}_{\mathrm{g}}\right)$ of the membranes. $\mathrm{T}_{\mathrm{g}}$ is a measure of the degree of rigidity of the polymer chain. The procedure for the testing was adopted as per reported literature [35]. (Model: Mettler Toledo DSC 822e). The membrane samples were weighed and heated between a temperature range of $30-400^{\circ} \mathrm{C}$ at a heating rate of $10^{\circ} \mathrm{C} \mathrm{min}^{-1}$ to remove the thermal history and were cooled thereafter from $400^{\circ} \mathrm{C}-30^{\circ} \mathrm{C}$ at a cooling rate of $10^{\circ} \mathrm{C} \mathrm{min}^{-1}$. The heating cycle was then repeated as earlier and $\mathrm{T}_{\mathrm{g}}$ of the sample was determined.

\subsection{Filtration Studies}

\subsubsection{Pure Water Flux (PWF) studies}

Filtration experiments were performed using self fabricated cross-flow filtration cell as shown in Fig.3. Membrane modules were prepared by 'potting' the ends of a stainless steel pipe with epoxy adhesive and hardener. Each membrane module comprised of 10 hollow fibers of $20 \mathrm{~cm}$ length.Membranes were initially compacted at trans-membrane pressure (TMP) of 2 bar for 30 minutes before proceeding with the flux measurements. PWF was calculated using the following equation,

$$
J_{w}=\frac{Q}{(\Delta t \cdot A)}
$$

Where, $J_{w}\left(\mathrm{~L} \cdot \mathrm{m}^{-2} \cdot \mathrm{h}^{-1}\right)$ is the pure water flux, and $Q(\mathrm{~L})$ is the amount of water collected for $\Delta t$ (h) time duration using a membrane of area $A\left(\mathrm{~m}^{2}\right)$.

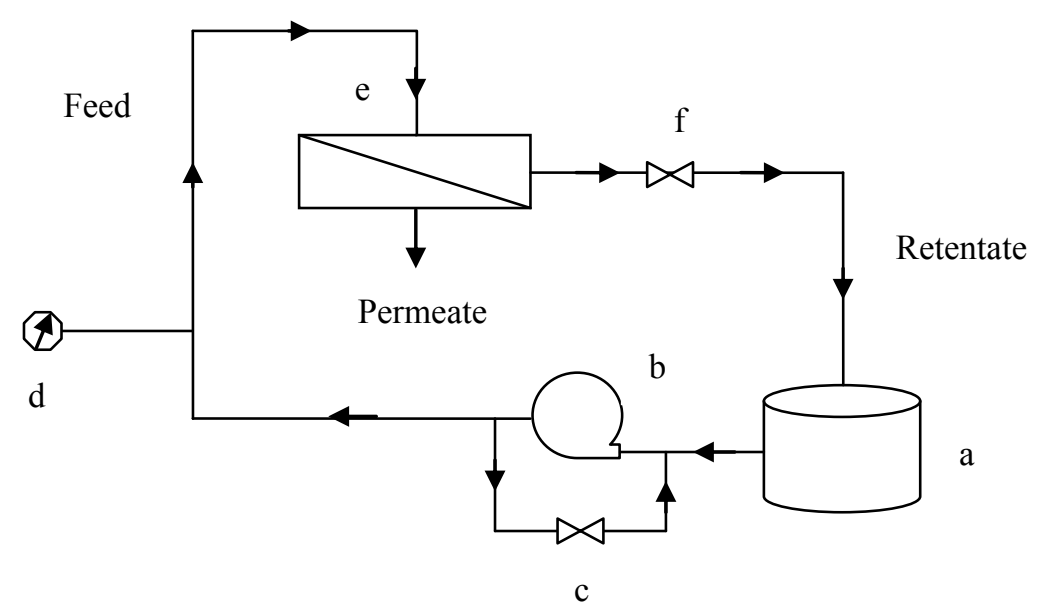


Fig.3: Self-Fabricated cross-flow filtration setup (a) Feed tank, (b) Diaphragm pump (c) Bypass valve (d) Pressure Guage (e) Membrane module (f) Retentate Flow-control valve

\subsubsection{Hazardous Dye rejection studies}

The dye rejection performances of all hollow fiber membranes were studied using the crossflow filtration setup. $300 \mathrm{ppm}$ of RR120 dye in aqueous solution was used as feed. The rejection studies were conducted at a constant pressure of 2 bar and permeate was collected for a known interval of time. The absorbance of RR120 dye was measured at $535 \mathrm{~nm}$ wavelength with a Hitachi U5000 spectrophotometer. Then the percentage rejection was calculated using the following equation,

$$
\% R=\left(1-\frac{C_{p}}{C_{f}}\right) \times 100
$$

where, $\% R=$ rejection $(\%), C_{p}$ and $C_{f}$ are the concentrations $(\mathrm{mM})$ of permeate and feed solutions, respectively.

Since concentration is directly proportional to the measured absorbance, we can also calculate rejection using the following equation,

$$
\% R=\left(1-\frac{A_{p}}{A_{f}}\right) \times 100
$$

where, $\% R=$ Rejection $(\%), A_{p}$ and $A_{f}$ are the Absorbance values of permeate and feed solutions, respectively.

\subsubsection{Antifouling studies}

To evaluate the antifouling property of the membrane, after dye filtration for 60 minutes, the membrane was subjected to pressurized cleaning with pure water atincreased pressure of 4 bar for 10 minutes and then the flux of the membrane $J_{c}$, was measured according to the method described earlier in section 2.7.1 at a pressure of 2 bar. The flux recovery ratio was calculated using the following equation,

$$
F R R=\left(\frac{J_{c}}{J_{w}}\right) \times 100
$$

Where, FRR is flux recovery ratio $(\%), J_{w}$ and $J_{c}$ are the pure water flux $\left(\mathrm{L} \cdot \mathrm{m}^{-2} \cdot \mathrm{h}^{-1}\right)$ and flux after cleaning $\left(\mathrm{L} \cdot \mathrm{m}^{-2} \cdot \mathrm{h}^{-1}\right)$ of the membrane, respectively.

\section{Results and Discussion}

\subsection{Synthesis of PANI-nanofibers}


PANI-nanofibers were synthesized by interfacial polymerization of aniline monomer in acidic medium. Standard chemical oxidation polymerization of aniline yields agglomerated PANI without nanofiber morphology. In the interfacial polymerization technique, the polymerization reaction takes place at the interface of the two immiscible liquids [28]. The vigorous stirring results in the dispersion of o-xylene as micro droplets increasing the area available for interfacial polymerization. Nanofiber size and yield was affected by the mole ratio of oxidant to aniline [33]. As observed in SEM and TEM images and shown in Fig.4, the PANI-nanofiber diameters were in the range of $60-80 \mathrm{~nm}$ and length was around $150-$ $300 \mathrm{~nm}$. PANI-nanofibers are hydrophilic in nature and hence increase the membrane wettability and subsequently the water flux when blending with polymer membranes [30].

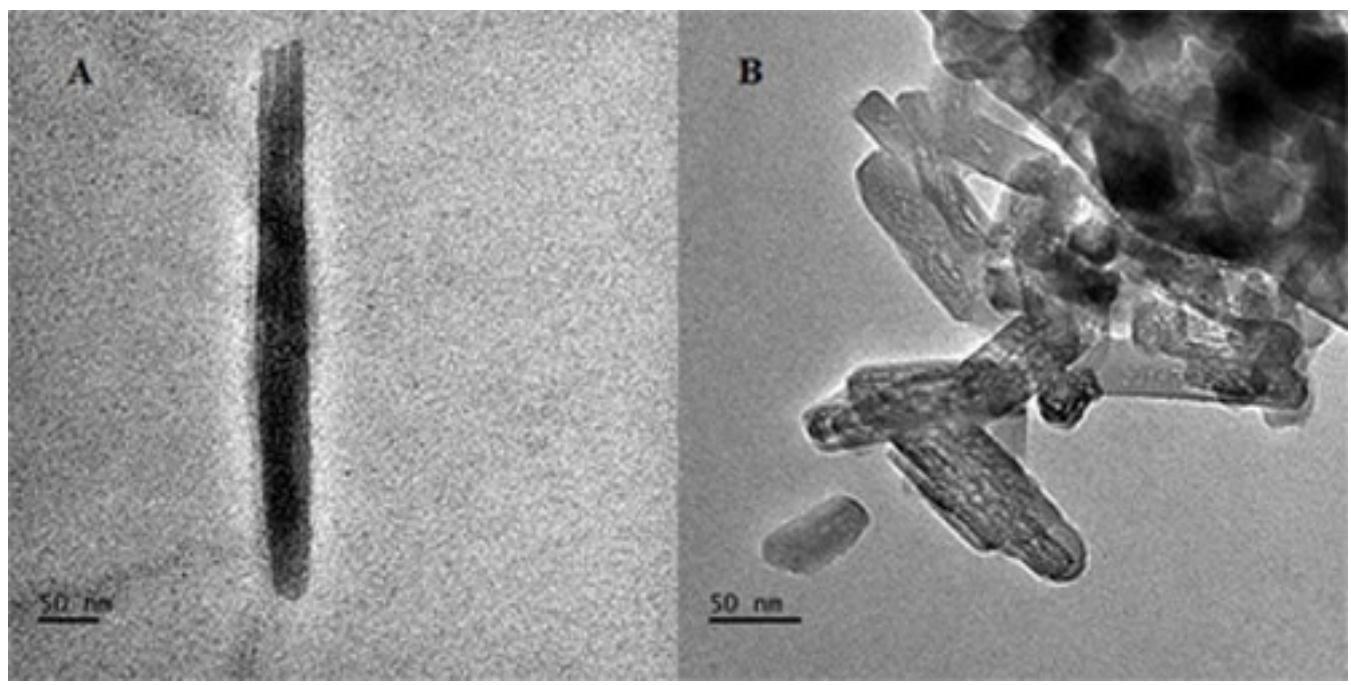

Fig. 4. TEM images of PANI coated $\mathrm{TiO}_{2}$ nanotubes (A and $\mathrm{B}$ )

\subsection{Membrane Morphology}

The addition of PVP and PANI-nanofibers greatly increased the viscosity of the casting dope solution. The addition of PVP may have resulted in reduced interaction between PSf and NMP thereby reducing the solvating power of NMP [36]. Also, the increase in viscosity due to the addition of PANI-nanofibers may be due to its large chain dimension, which was easy to aggregate and its ability to gather NMP due to the high surface energy, which weakened PSf and NMP interaction [36]. Thus, when increase of PANI-nanofiber concentration to 1.0 wt $\%$ of PSf, it was difficult to disperse them evenly in the casting dope solution and it clogged the spinneret during the hollow fiber spinning process.

From the SEM images of the membrane surface shown in Fig.5, it is observed that, the membrane is having morphology with asymmetric pores and non-uniform distribution of pores. The cross section image showed that, the membranes have well formed asymmetrical 
structure with dense top layers and porous sub-layer. There were well-formed finger like pores of almost equal length, with interconnected macrovoids below both outer and inner surfaces demarked by a thin polymer layer. PANI-nanofibers have high surface energy and hydrophilicity, thus when the hollow fiber membrane was immersed in a water bath, the PANI-nanofibers may migrate from the polymer matrix towards the water bath so as to reduce the interfacial energy between the two phases [30]. The migration would leave cavities in the polymer matrix, which in turn would increase the membrane porosity and have interconnection between the finger like pores and macrovoids. This agrees with research of Fan et al. [30], who reported higher membrane porosity and better interconnected pores in PSf/PANI-nanocomposite membrane compared to the neat PSf membrane. The PANInanofibers aggregate on the polymer-water interface skin layer leading to the dense top layer morphology and increases the membrane surface hydrophilicity. The membrane had a porous internal structure, which may be due to the addition of PVP in the polymer dope. PVP is a water-soluble polymer and hence most of it may be leached out from the polymer matrix when the membrane is immersed into water bath and these sites become micro pores [36]. Also, there is an increase in the number of pores on the membrane surface due to PVP additive. The increase in pores may be due to increased viscosity of the casting solution and a reduction in the miscibility of the casting solution with water, which may increase the ratio of water inflow to solvent outflow resulting in a more porous membrane [31] [36]. Simultaneously, there is also a reduction in the size of the pores due to additives. The higher molecular weight additives may take longer time to reach the membrane surface during the membrane formation process which will give sufficient time for the polymer molecules to aggregate on top of it and form a denser skin layer with the relatively smaller size pores [31]. However, Fan et al. [30] have reported a slight increase in the pore size due to the addition of PANI-nanofibers. Zhao et al. [36] found that, addition of PVP and PANI-nanofibers decreased the thermodynamic stability of the casting solution thus enhancing demixing of the casting solution. It was also reported that, there was a decrease of water intake during preparation leading to improvement in membrane structure and performance. 

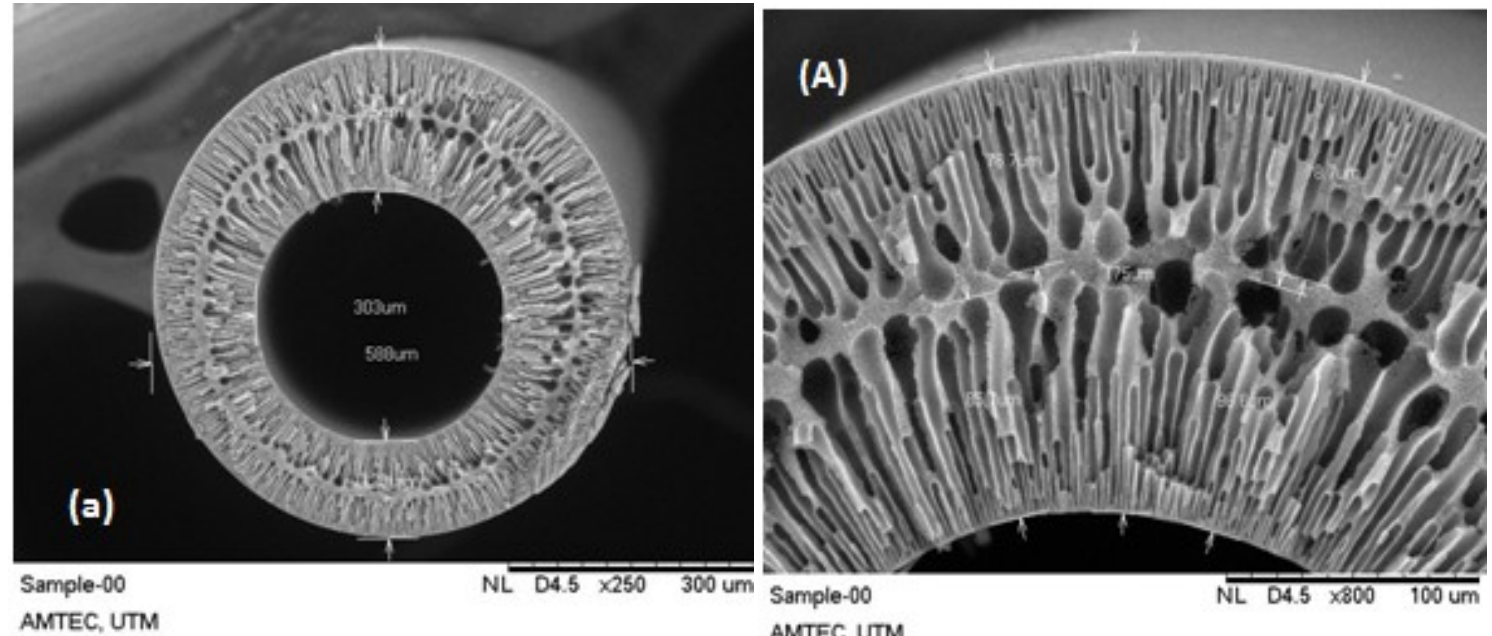

NL $\overline{04.5 \times 250 \quad 300 \mathrm{um}}$

AMTEC, UTM

AMTEC, UTM
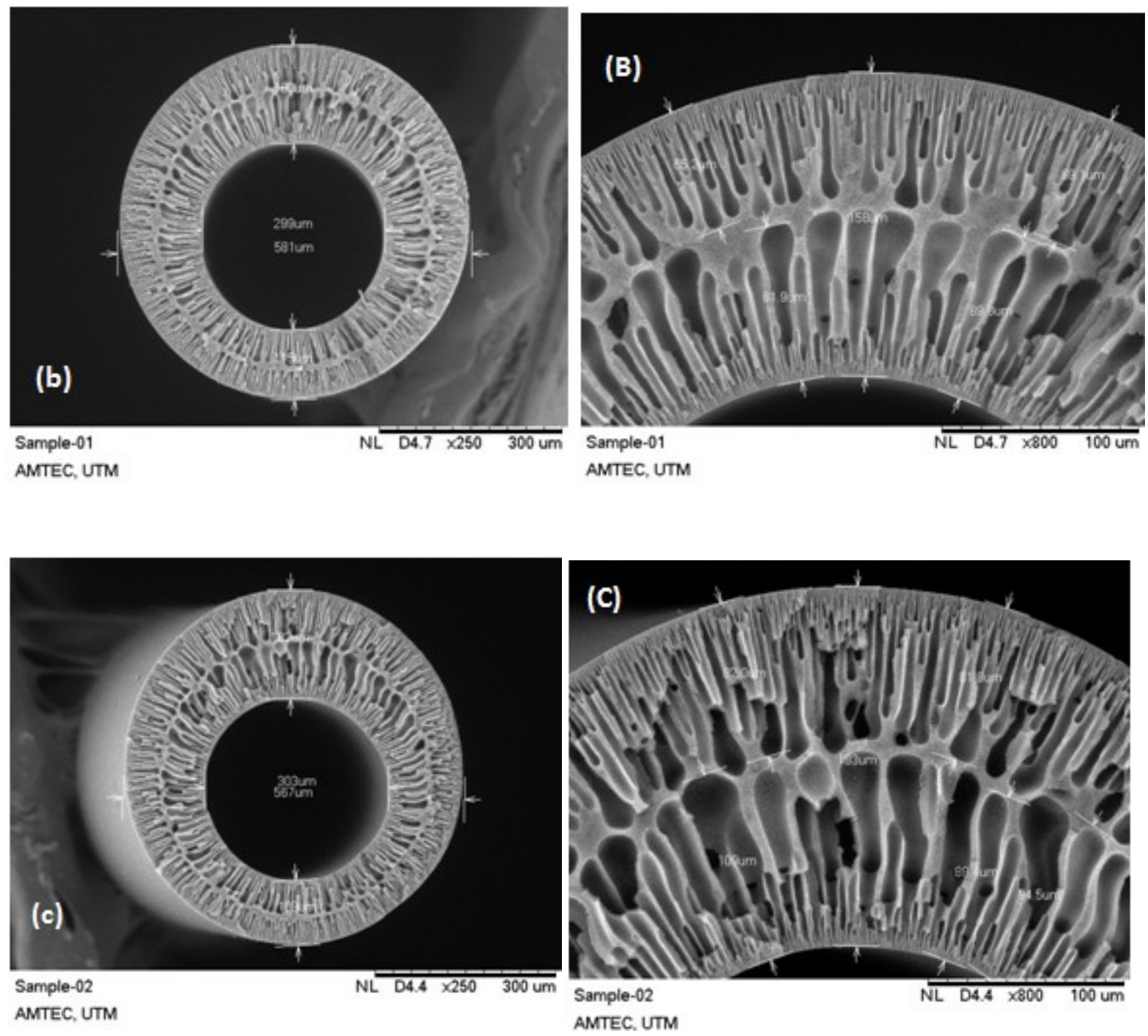

Fig.5: SEM images of PSf/PVP/PANI-nanofiber hollow fiber membrane cross-sections. (a,

A) Sample-00: M0, (b,B) Sample-01: M0.25, (c,C) Sample-02: M0.5.

\subsection{Contact Angle}


Contact Angle is a good indication of hydrophilic property of the material. The static contact angles for the new membranes measured by the sessile drop method are reported in Fig.6. From the figure it is clear that, the contact angle reduces with increasing concentration of PANI-nanofiber indicating an increase in the hydrophilicity of the membrane. As discussed in section 3.2, this may be due to PANI-nanofibers, which migrate towards the polymer-water interface and aggregate at the membrane surface [36]. The PANI-nanofibers being highly hydrophilic in nature may be responsible for reducing the water contact angle on the membrane surface.

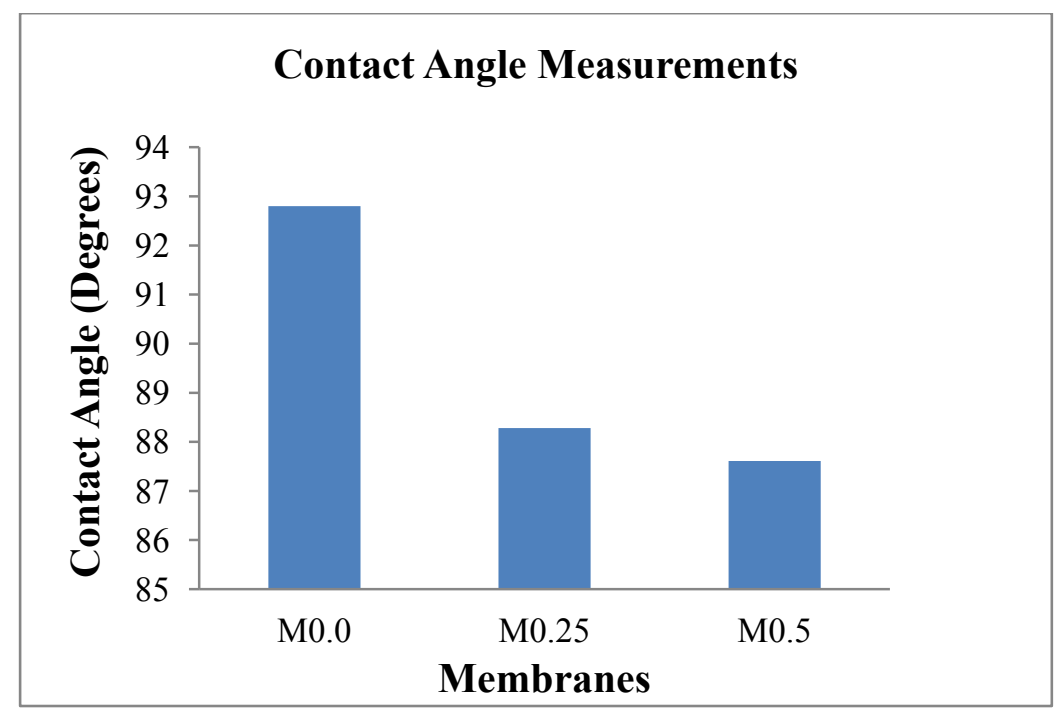

Fig.6: Contact angle measurements of PSf/PVP/PANI-nanofiber hollow fiber membranes.

\subsection{Zeta Potential measurements}

The different ionisable groups in the membrane material affect the zeta potential values of the membrane for range of $\mathrm{pH}$. Zeta potentials calculated based on measurements of streaming potential of the hollow fiber membranes between $\mathrm{pH}$ ranges of 2.0-7.0 are shown in Fig.7. The $\mathrm{pH}$ dependent measurements enable us to identify the isoelectric point of the membranes [37]. The isoelectric point is the $\mathrm{pH}$ value when there is no charge present on the membrane surface. From the graph, we can observe that the isoelectric point of the membranes shift towards lower $\mathrm{pH}$ with increasing PANI-nanofiber concentration and that the membrane surface has a negative charge over almost the entire $\mathrm{pH}$ range tested. This is a qualitative indicator of electrostatic contribution to the rejection of dyes [20]. 


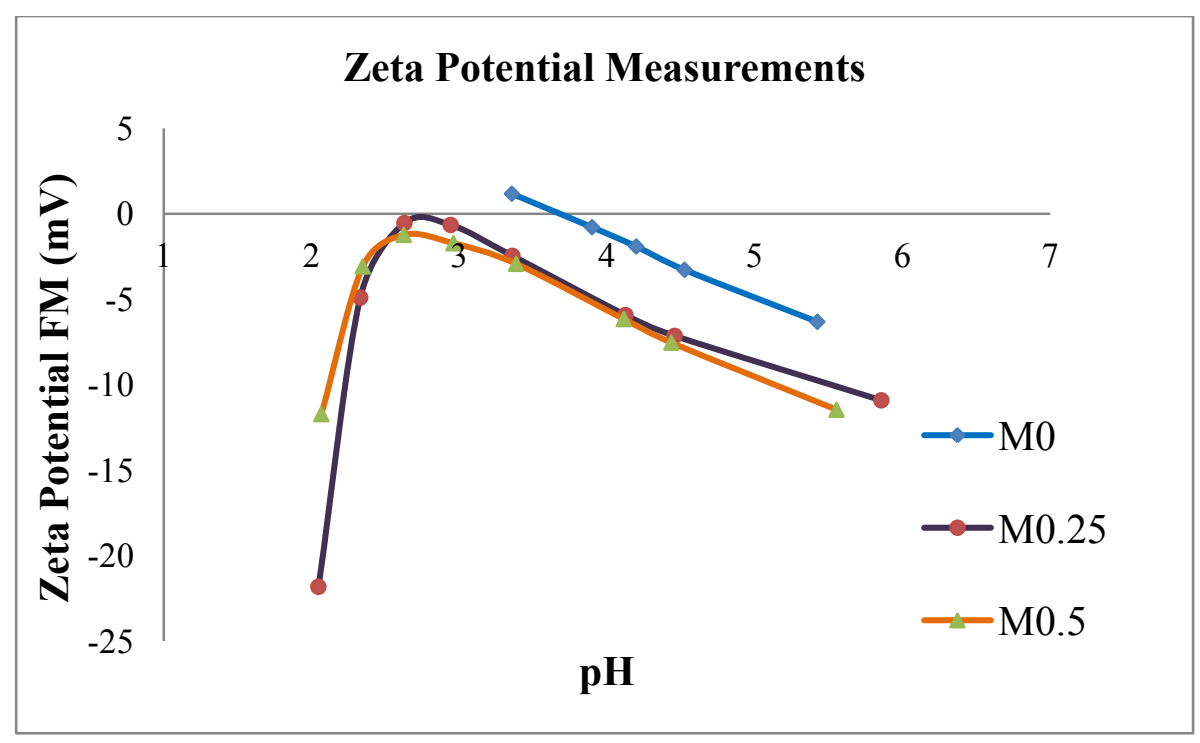

Fig.7: Zeta Potential measurements of PSf/PVP/PANI-nanofiber hollow fiber membranes.

\subsection{Differential Scanning Calorimetry (DSC) studies}

DSC graphs for the new membranes are shown in Fig.8. The glass transition temperature $\left(\mathrm{T}_{\mathrm{g}}\right)$ of the membranes increase with increasing concentration of PANI-nanofibers and thus have higher thermal resistance [38]. Usually the dyeing and rinsing procedures on textiles are conducted at temperatures higher than room temperature and hence higher temperature wastewater is generated [7] [35]. Thus one can conclude that, the membrane has better resistance for treating higher temperature dye wastewater.

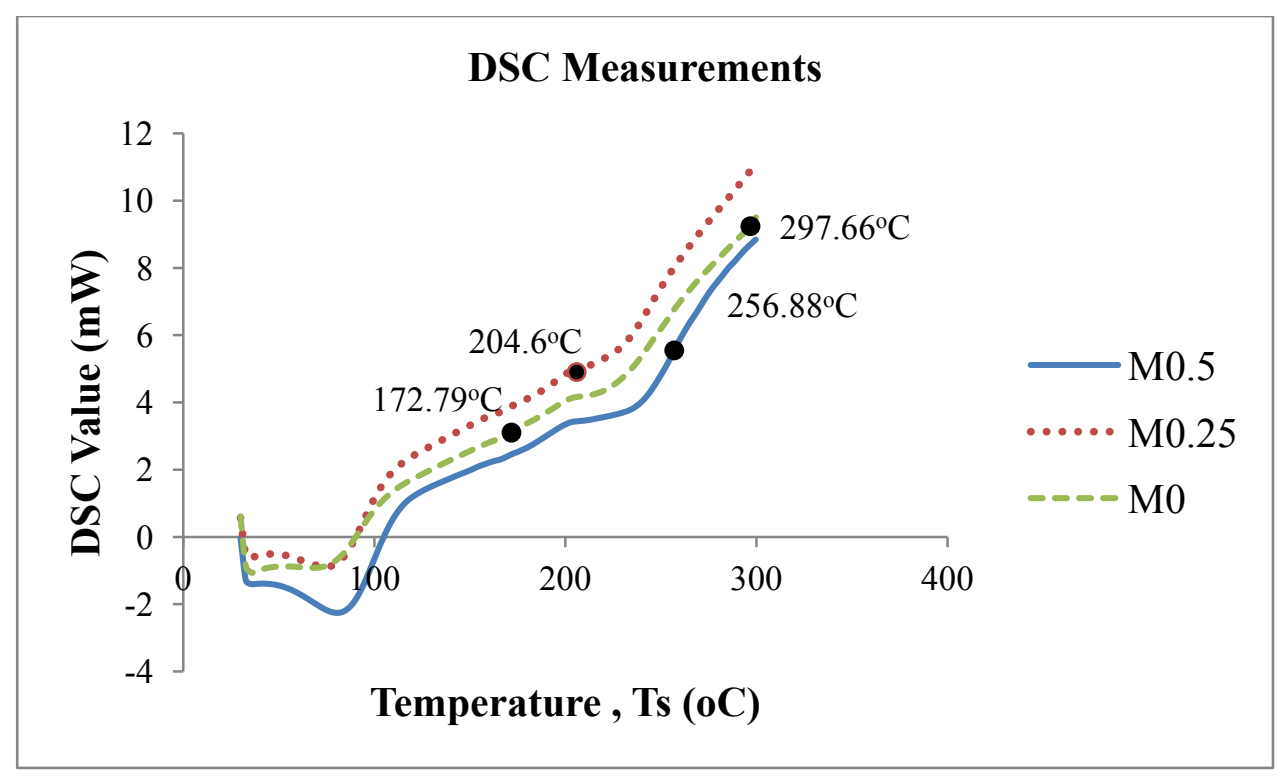

Fig.8: DSC thermographs of PSf/PVP/PANI-nanofiber hollow fiber membranes.

3.5 Pure Water Flux (PWF) Studies 
Hollow fiber membranes show increased water flux when compared to flat sheet membranes since they have a higher surface area per unit of membrane module volume. The membrane cross-flow filtration tests indicate that, flux increases with increasing PANInanofiber concentration for constant pressure of 2 bar as shown in Fig.9. The higher water flux for new membranes can be attributed to increased hydrophilicity and increased porosity due to PVP and PANI-nanofiber additives. The nanocomposite membrane showed increased flux and rejection due to the additives. The PANI-nanofibers are hydrophilic in nature and during the membrane formation process, they migrate towards the surface of the membrane [30]. This migration through the polymer matrix, forms finger like interconnected pores in the membrane cross section. This increases flux and reduces resistance to flow of water through the membrane Also, due to the hydrophilic PANI-nanofibers moving to the surface, the membrane surface becomes more hydrophilic and this in turn increases wetting of the surface contributing to the increase in flux. PVP has been used as a pore forming agent and is reported to increase the formation of pores on the membrane surface [31] thus increasing flux of water through the membrane. Also, since PVP is a water-soluble polymer, it may be leached out from the polymer matrix when the membrane is immersed in water, causing the formation of micro voids and thereby increasing the porosity of the membrane [36].

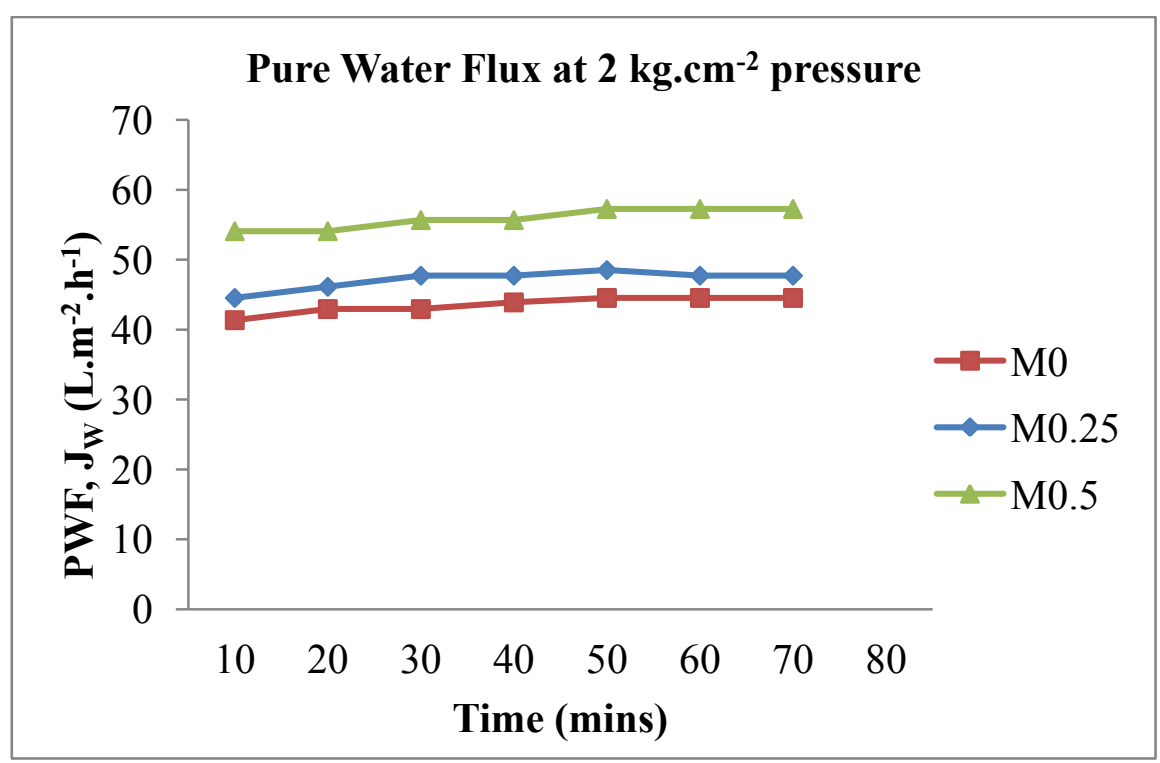

Fig.9: PWF of PSf/PVP/PANI nanofiber hollow fiber membrane.

\subsection{Hazardous Dye rejection and Flux Recovery Studies}

The new membranes showed very good rejection for RR120 dye at 2 bar pressure. The \% rejection of dyeincreases with increase in PANI-nanofiber concentration as shown in Fig.10 
with a maximum value of $99.25 \%$ for M0.5 membrane. The new membrane shows increased dye rejection due to the additives and this can be attributed to 1) sieving mechanism due to reduced pore size, 2) electrostatic repulsion due to the residual negative charge on the membrane surface and 3) adsorption of the dye on the membrane surface. As observed, increase in PANI-nanofibers lead to increase in all of the above three rejection mechanisms mentioned above and result in increased dye rejection by the new membrane.

During the course of dye filtration, fouling is unavoidable [7] and hence the membrane flux reduces over a period of time. The decrease in flux may be attributed to dye adsorption on the membrane surface and pores. To evaluate the cleaning effectiveness and the ability of the membrane to recover flux after being fouled, it is subjected to cleaning with distilled water at increased pressure of 4 bar for 10 minutes and afterwards the pure water flux at 2 bar was again measured. Flux recovery ratio (FRR), which is a measure of the antifouling property of the membrane, was calculated and reported in Fig.11. From the graphs, it is clear that increasing PANI-nanofiber concentration resulted in better flux recovery ratio. The presence of PANI-nanofibers on the surface skin layer results in increased hydrophilicity of the membrane, which may be responsible for the increased antifouling ability of the membrane. Hydrophilic surface may not be easily adsorbed by the dye molecules and thereby improves the antifouling property [36]. Thus we can conclude that the addition of PANI-nanofibers imparts the better antifouling ability to the membranes.

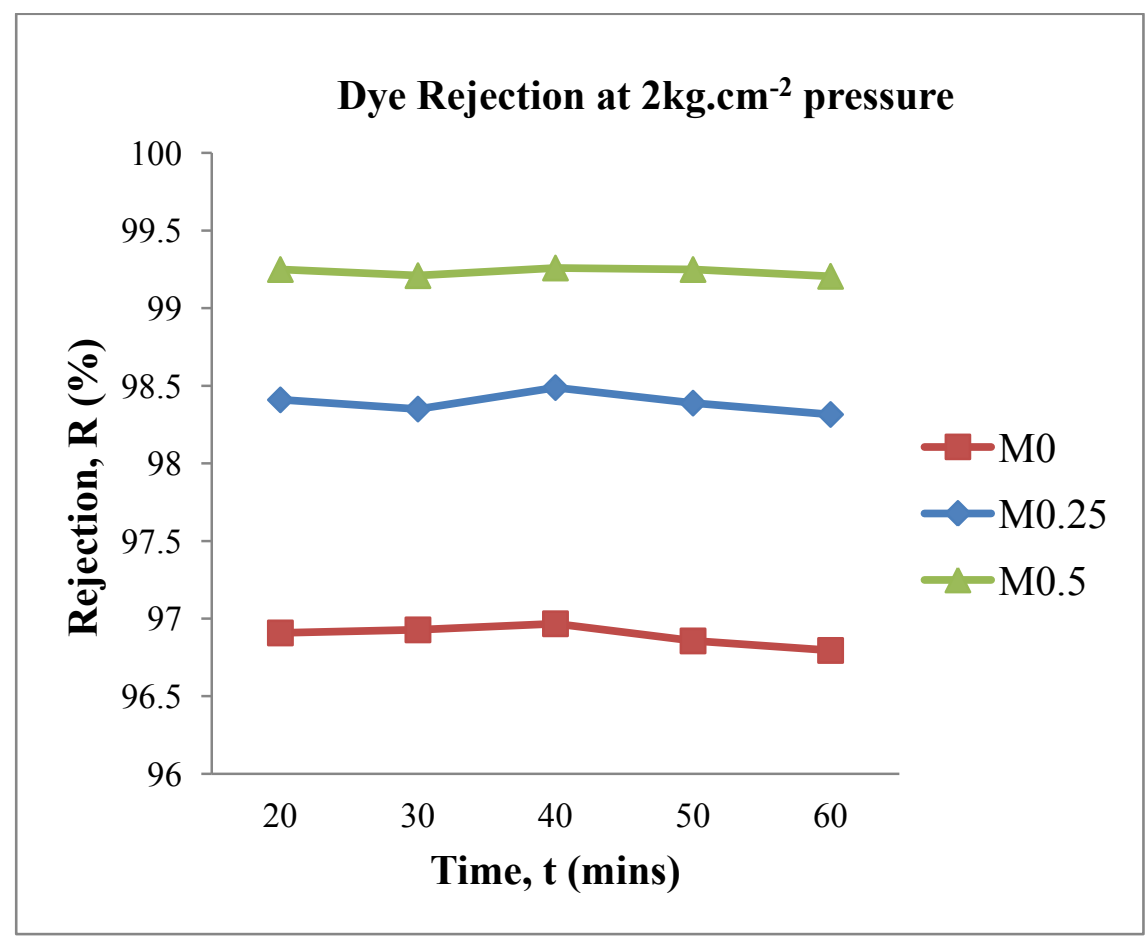


Fig.10: Performance of PSf/PVP/PANI-nanofiber membrane for rejection of RR120 dye.

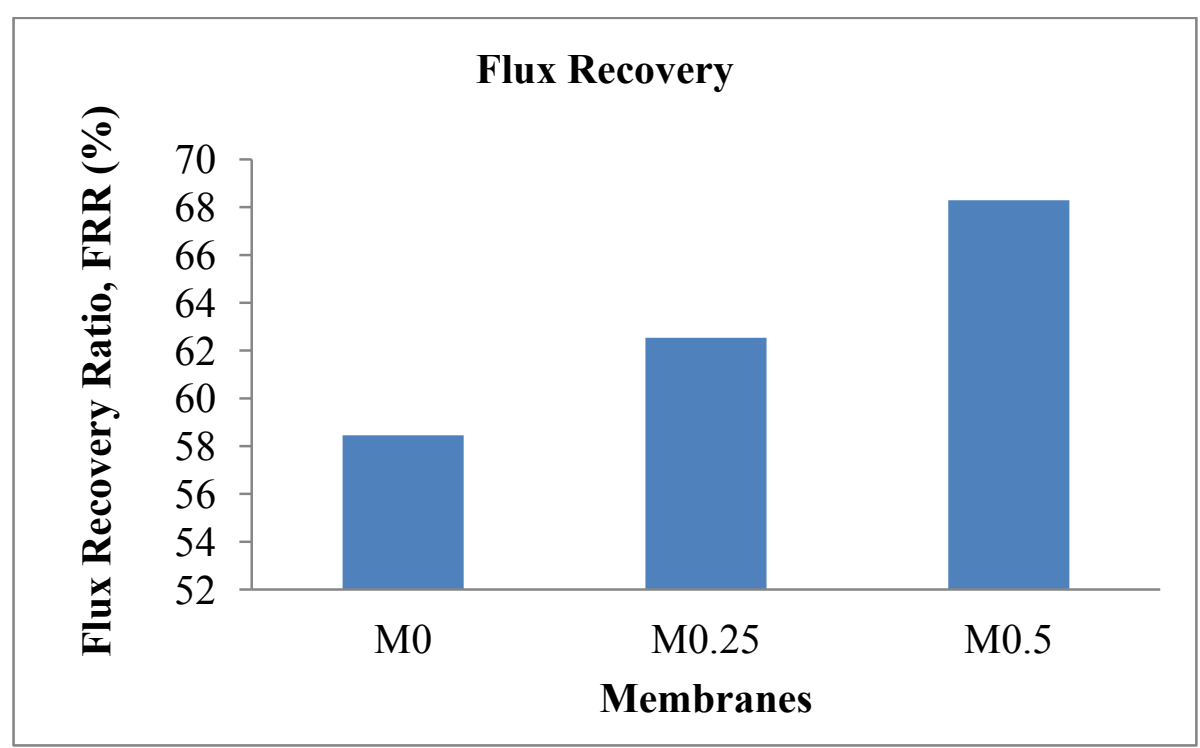

Fig.11: Flux Recovery Ratio of PSf/PVP/PANI nanofiber membrane for RR120 dye.

\section{Conclusions}

The effects of PANI-nanofibers on the hollow fiber membrane structure and performance were analysed and reported. Filtration experiments were conducted to assess the applicability and performance of the membrane for rejection of RR120 hazardous dye from water. From the results obtained, the following can be concluded.

1. PANI-nanofibers in the range of $60-80 \mathrm{~nm}$ and length around $150-300 \mathrm{~nm}$ were synthesized via. interfacial polymerization process by oxidation of aniline in acidic medium.

2. Novel PSf/PVP/PANI-nanofiber nanocomposite ultrafiltration hollow fiber membranes were prepared via dry-wet spinning technique. The novel membranes showed well formed asymmetrical structure with dense top layers and porous sub-layer.

3. The addition of PANI-nanofibers has resulted in a membrane with interconnected finger like porous structure and more hydrophilic surface. The addition of PVP has resulted in micro pores within the membrane structure with increased permeability.

4. The contact angle of the membranes decreased with increasing PANI-nanofiber concentration, indicating increased hydrophilicity of the new membranes. The new membranes also display higher thermal resistance. 
5. The PWF and rejection studies indicated improvement in membrane flux and dye rejection with increasing PANI-nanofiber concentration.The best results were obtained for M0.5 membrane with maximum rejection $99.25 \%$ at 2 bar for RR120 dye.

6. The new membranes have increased antifouling property due to the addition of PANInanofibers.

\section{Acknowledgements}

We thank The Director, NITK, Surathkal, India for supporting research work and providing financial assistance for travelling to Malaysia. We also thank members of Advanced Membrane TEchnology Research Centre (AMTEC), Universiti Teknologi Malaysia, 81310 UTM, Skudai, Johor, Malaysia for their help.

\section{References}

[1] V.K. Gupta, R. Kumar, A. Nayak, T.A. Saleh, M. Barakat, Adsorptive removal of dyes from aqueous solution onto carbon nanotubes: A review, Adv. Colloid Interface Sci., 193 (2013) 24-34.

[2] M.T. Yagub, T.K. Sen, S. Afroze, H. Ang, Dye and its removal from aqueous solution by adsorption: A review, Adv. Colloid Interface Sci., 209 (2014) 172-184.

[3] M. Rafatullah, O. Sulaiman, R. Hashim, A. Ahmad, Adsorption of methylene blue on low-cost adsorbents: a review, J. Hazard. Mater., 177 (2010) 70-80.

[4] A.K. Verma, R.R. Dash, P. Bhunia, A review on chemical coagulation/flocculation technologies for removal of colour from textile wastewaters, J. Environ. Manage, 93 (2012) 154-168.

[5] P.V. Nidheesh, R. Gandhimathi, S.T. Ramesh, Degradation of dyes from aqueous solution by Fenton processes: a review, Environ. Sci. Pollut. Res, 20 (2013) 2099-2132.

[6] F. Zhang, A. Yediler, X. Liang, A. Kettrup, Effects of dye additives on the ozonation process and oxidation by-products: a comparative study using hydrolyzed $\mathrm{CI}<\mathrm{i}>$ Reactive Red 120</i>, Dyes Pigm., 60 (2004) 1-7.

[7] Y.K. Ong, F.Y. Li, S.-P. Sun, B.-W. Zhao, C.-Z. Liang, T.-S. Chung, Nanofiltration hollow fiber membranes for textile wastewater treatment: Lab-scale and pilot-scale studies, Chem. Eng. Sci., 114 (2014) 51-57.

[8] S. Yu, Z. Chen, Q. Cheng, Z. Lü, M. Liu, C. Gao, Application of thin-film composite hollow fiber membrane to submerged nanofiltration of anionic dye aqueous solutions, Sep. Purif. Technol., 88 (2012) 121-129. 
[9] X. Wei, X. Kong, C. Sun, J. Chen, Characterization and application of a thin-film composite nanofiltration hollow fiber membrane for dye desalination and concentration, Chem. Eng. J. (Lausanne), 223 (2013) 172-182.

[10] Y. Zheng, G. Yao, Q. Cheng, S. Yu, M. Liu, C. Gao, Positively charged thin-film composite hollow fiber nanofiltration membrane for the removal of cationic dyes through submerged filtration, Desalination, 328 (2013) 42-50.

[11] S. Maurya, K. Parashuram, P. Singh, P. Ray, A. Reddy, Preparation of polysulfonepolyamide thin film composite hollow fiber nanofiltration membranes and their performance in the treatment of aqueous dye solutions, Desalination, 304 (2012) 11-19.

[12] M.D. Afonso, R. Bórquez, Review of the treatment of seafood processing wastewaters and recovery of proteins therein by membrane separation processes-prospects of the ultrafiltration of wastewaters from the fish meal industry, Desalination, 142 (2002) 29-45.

[13] N. Hamid, A.F. Ismail, T. Matsuura, A. Zularisam, W. Lau, E. Yuliwati, M. Abdullah, Morphological and separation performance study of polysulfone/titanium dioxide (PSF/TiO $<$ sub $>2</$ sub $>$ ) ultrafiltration membranes for humic acid removal, Desalination, 273 (2011) $85-92$.

[14] E. Yuliwati, A.F. Ismail, T. Matsuura, M.A. Kassim, M. Abdullah, Effect of modified PVDF hollow fiber submerged ultrafiltration membrane for refinery wastewater treatment, Desalination, 283 (2011) 214-220.

[15] Allègre, C., Moulin, P., Maisseu, M., Charbit, F., "Treatment and reuse of reactive dyeing effluents", Journal of Membrane Science, 269 (2006), 15-34.

[16] Allègre, C., Moulin, P., Maisseu, M., Charbit, F., "Savings and re-use of salts and water present in dye house effluents", Desalination, 162 (2004), 13-22.

[17] Ranganathan, K., Karunagaran, K., Sharma, D.C., "Recycling of wastewaters of textile dyeing industries using advanced treatment technology and cost analysis - Case studies", Resources, Conservation and Recycling, 50 (2007), 3, 306-318.

[18] Reife, A., Freeman, H.S., "Environmental Chemistry of Dyes and Pigments", John Wiley \& Sons, (1996).

[19] Brandon, C.A., "Closed Cycle Textile Dyeing: Full-scale Hyperfiltration Demonstration, Demonstration (Design), U.S. Environmental Protection Agency, EPA-600/2-80-005, March 1980.

[20] S.R. Panda, S. De, Preparation, characterization and performance of $\mathrm{ZnCl}<\mathrm{sub}>$ $2</$ sub $>$ incorporated polysulfone (PSF)/polyethylene glycol (PEG) blend low pressure nanofiltration membranes, Desalination, 347 (2014) 52-65. 
[21] R. Kumar, A.M. Isloor, A. Ismail, T. Matsuura, Performance improvement of polysulfone ultrafiltration membrane using $\mathrm{N}$-succinyl chitosan as additive, Desalination, 318 (2013) 1-8.

[22] R. Kumar, A.M. Isloor, A.F. Ismail, T. Matsuura, Synthesis and characterization of novel water soluble derivative of Chitosan as an additive for polysulfone ultrafiltration membrane, J. Membr. Sci., 440 (2013) 140-147.

[23] M. Padaki, A.M. Isloor, R. Kumar, A. Fauzi Ismail, T. Matsuura, Synthesis, characterization and desalination study of composite NF membranes of novel Poly[(4aminophenyl)sulfonyl]butanediamide (PASB) and methyalated Poly[(4aminophenyl)sulfonyl]butanediamide (mPASB) with Polysulfone (PSf), J. Membr. Sci., 428 (2013) 489-497.

[24] R. Kumar, A.M. Isloor, A.F. Ismail, S.A. Rashid, A.A. Ahmed, Permeation, antifouling and desalination performance of $\mathrm{TiO} 2$ nanotube incorporated $\mathrm{PSf} / \mathrm{CS}$ blend membranes, Desalination, 316 (2013) 76-84.

[25] X. Qu, P.J. Alvarez, Q. Li, Applications of nanotechnology in water and wastewater treatment, Water Res., 47 (2013) 3931-3946.

[26] Razmjou, A., Resosudarmo, A., Holmes, R.L., Li, H., Mansouri, J., Chen, V., "The effect of modified $\mathrm{TiO}_{2}$ nanoparticles on the polyethersulfone ultrafiltration hollow fiber membranes", Desalination, 287 (2012), 271-280.

[27] Jeong, B., Hoek, E.M.V., Yan, Y., Subramani, A., Huang, X., Hurwitz, G., Ghosh, A.K., Jawor, A., "Interfacial polymerization of thin film nanocomposites: A new concept for reverse osmosis membranes", Journal of Membrane Science, 294 (2007), 1-7.

[28] S. Bhadra, D. Khastgir, N.K. Singha, J.H. Lee, Progress in preparation, processing and applications of polyaniline, Prog. Polym. Sci., 34 (2009) 783-810.

[29] Huang, J., "Synthesis and applications of conducting polymer polyanlinenanofibers", Pure and Applied Chemistry, 78 (2006), 15-27.

[30] Z. Fan, Z. Wang, N. Sun, J. Wang, S. Wang, Performance improvement of polysulfone ultrafiltration membrane by blending with polyaniline nanofibers, J. Membr. Sci., 320 (2008) 363-371.

[31] B. Chakrabarty, A. Ghoshal, M. Purkait, Preparation, characterization and performance studies of polysulfone membranes using PVP as an additive, J. Membr. Sci., 315 (2008) 3647. 
[32] C. Feng, K. Khulbe, T. Matsuura, A. Ismail, Recent progresses in polymeric hollow fiber membrane preparation, characterization and applications, Sep. Purif. Technol., 111 (2013) 43-71.

[33] S. Xing, H. Zheng, G. Zhao, Preparation of polyaniline nanofibers via a novel interfacial polymerization method, Synth. Met., 158 (2008) 59-63.

[34] C. Ong, W. Lau, P. Goh, B. Ng, A. Ismail, Preparation and characterization of PVDFPVP-TiO2 composite hollow fiber membranes for oily wastewater treatment using submerged membrane system, Desalin. Water Treat, (2013) 1-11.

[35] A. Zulhairun, A. Ismail, T. Matsuura, M. Abdullah, A. Mustafa, Asymmetric mixed matrix membrane incorporating organically modified clay particle for gas separation, Chem. Eng. J. (Lausanne), 241 (2014) 495-503.

[36] S. Zhao, Z. Wang, X. Wei, X. Tian, J. Wang, S. Yang, S. Wang, Comparison study of the effect of PVP and PANI nanofibers additives on membrane formation mechanism, structure and performance, J. Membr. Sci., 385 (2011) 110-122.

[37] E. Alventosa-deLara, S. Barredo-Damas, E. Zuriaga-Agustí, M. Alcaina-Miranda, M. Iborra-Clar, Ultrafiltration ceramic membrane performance during the treatment of model solutions containing dye and salt, Sep. Purif. Technol., 129 (2014) 96-105.

[38] A. Rahimpour, S. Madaeni, A. Taheri, Y. Mansourpanah, Coupling $\mathrm{TiO}<\mathrm{sub}>2</$ sub $>$ nanoparticles with UV irradiation for modification of polyethersulfone ultrafiltration membranes, J. Membr. Sci., 313 (2008) 158-169.

\section{List of Contents}

Fig.1: Molecular Structure of Reactive Red 120 dye.

Fig.2:(a) Schematic representation of the hollow fiber spinning set up, (b) cross-section of the spinneret during spinning [32].

Fig.3: Self-Fabricated cross-flow filtration setup (a) Feed tank, (b) Diaphragm pump (c) Bypass valve (d) Pressure Guage (e) Membrane module (f) Retentate Flow-control valve.

Fig.4: TEM images of PANI coated $\mathrm{TiO}_{2}$ nanotubes (A and B)

Fig.5:SEM images of PSf/PVP/PANI-nanofiber hollow fiber membrane cross-sections. (a,

A) Sample-00: M0, (b,B) Sample-01: M0.25, (c,C) Sample-02: M0.5.

Fig.6:Contact angle measurements of PSf/PVP/PANI-nanofiber hollow fiber membranes.

Fig.7: Zeta Potential measurements of PSf/PVP/PANI-nanofiber hollow fiber membranes.

Fig.8: DSC thermographs of PSf/PVP/PANI-nanofiber hollow fiber membranes. 
Fig.9:PWF of PSf/PVP/PANI nanofiber hollow fiber membrane.

Fig.10:Performance of PSf/PVP/PANI-nanofiber membrane for rejection of RR120 dye.

Fig.11:Flux Recovery Ratio of PSf/PVP/PANI nanofiber membrane for RR120 dye.

\section{List of Tables}

Table1: Dope solutions for PSf/PVP/PANI Nanofiber hollow fiber membrane

Table 2: Spinning Parameters for PSf/PVP/PANI-nanofiber hollow fiber membranes 